\title{
Histone deacetylase 3 promotes pancreatic cancer cell proliferation, invasion and increases drug-resistance through histone modification of P27, P53 and Bax
}

\author{
FENG JIAO $^{1 *}$, HAI HU ${ }^{1 *}$, CUNCUN YUAN ${ }^{2 *}$, ZILIANG JIN $^{1}$, ZHEN GUO $^{1}$, LIWEI WANG ${ }^{1}$ and LEI WANG ${ }^{1}$ \\ Departments of ${ }^{1}$ Oncology and ${ }^{2}$ Pathology, First People's Hospital, School of Medicine, \\ Shanghai Jiao Tong University, Shanghai 201620, P.R. China
}

Received May 22, 2014; Accepted July 7, 2014

DOI: $10.3892 /$ ijo.2014.2568

\begin{abstract}
Pancreatic cancer is one of the most aggressive solid malignancies with a dismal survival rate. Recent studies have shown that high expression levels of histone deacetylase 3 (HDAC3) correlate with malignant phenotype. However, the expression patterns and biological role of HDAC3 in pancreatic cancer remain unclear. In this study, our data showed that a higher level of HDAC3 protein expression was found in pancreatic cancer as compared to paired paracancerous tissues. Consistently, higher expression level of HDAC3 was found in all of the eight pancreatic cancer cell lines relative to human pancreatic ductal epithelial cells (HPDE). In addition, further function analysis revealed that HDAC3 can function as oncogenic protein, which could promote pancreatic cancer cell proliferation, migration and invasion, and may increase drug resistance. Moreover, the functional involvement of HDAC3 was partially correlated with post-induction repression of P53, P27 and Bax gene transcription, acting via H3K9 deacetylation. Taken together, our data suggest that HDAC3 participates in the pathogenesis and progression of pancreatic cancer through histone modification, which might be a pivotal epigenetic target against this devastating disease.
\end{abstract}

\section{Introduction}

Pancreatic cancer is a devastating disease with the worst prognosis among all the major human malignancies. Long-term

Correspondence to: Dr Li-Wei Wang or Dr Lei Wang, Department of Oncology, First People's Hospital, School of Medicine, Shanghai Jiao Tong University, 650 New Songjiang Road, Shanghai 201620, P.R. China

E-mail: yzwlw64@gmail.com

E-mail: wang_lei744@hotmail.com

*Contributed equally

Key words: pancreatic cancer, histone deacetylases 3, histone modification, cell invasion, drug resistance prognosis remains poor with a 5-year survival rate of $<5 \%$ after the initial diagnosis (1). One of the major hallmarks of pancreatic cancer is its extensive local tumor invasion and drug resistance, but the molecular events underlying this remain mysterious (2).

The alterations of the chromatin structure by histone acetylases (HATs) and histone deacetylases (HDACs) are involved in the regulation of gene transcription and also in the process of tumorigenesis. The deacetylation of lysine residues by HDACs can lead to chromatin compaction and gene inactivation (3). Recently studies showed that elevated levels of HDAC3 expression and activity caused epigenetic alterations associated with malignancies $(4,5)$. However, the role of HDAC3 in pancreatic cancer has not been well elucidated.

In this study, the potential effects of HDAC3 on pancreatic cancer were investigated. We found that HDAC3 was overexpressed in pancreatic cancer as compared to paired paracancerous tissues. Consistently, all of the eight pancreatic cancer cell lines had higher level of HDAC3 relative to human pancreatic ductal epithelial cells (HPDE). Further function analysis revealed that high HDAC3 expression could promote cancer cell proliferation, migration and invasion, and may increase drug resistance. Moreover, the functional involvement of HDAC3 was partially correlated with post-induction repression of P53, P27 and Bax gene transcription, acting via H3K9 deacetylation.

\section{Materials and methods}

Patients and tissue samples. Four cases of pancreatic cancer and paired paracancerous fresh tissues were obtained from Department of General Surgery, First People's Hospital, School of Medicine, Shanghai Jiao Tong University. For the use of all clinical materials for research purposes, prior written informed consent from all the patients and approval from the Ethics Committees of the First People's Hospital, School of Medicine, Shanghai Jiao Tong University were obtained.

Immunofluorescence. The experimental steps were performed as previous described (6). Pancreatic cancer tissue specimens stored at $-80^{\circ} \mathrm{C}$ were moved to $-20^{\circ} \mathrm{C}$ to equilibrate to the temperature of the cryostat. Sections were cut of the $7-8-\mu \mathrm{m}$ 
thickness, placed on slides and dried overnight at room temperature. Slides were then fixed by immersion in cold acetone $\left(-20^{\circ} \mathrm{C}\right)$ for $5 \mathrm{~min}$, air dried at room temperature and exposed to microwave for $15 \mathrm{~min}$. The prepared frozen tissue sections were either subjected to immunostaining or stored at $-20^{\circ} \mathrm{C}$.

The frozen tissue slices were re-fixed with paraformaldehyde at room temperature for $10 \mathrm{~min}$. After four rinses, the slices were blocked with 5\% BSA and 5\% goat serum for $60 \mathrm{~min}$ at $37^{\circ} \mathrm{C}$. Properly diluted primary antibody against HDAC3 (Abcam, dilution 1:2,500) was applied to frozen sections at $4^{\circ} \mathrm{C}$ overnight, followed by four rinses. The slides were stained with Dylight 546-conjugated goat anti-rabbit antibody (Jackson, dilution 1:100) for $1 \mathrm{~h}$ at room temperature. Finally, slides were stained with DAPI (Sigma) for $15 \mathrm{~min}$ and mounted in Antifade medium (Beyotime).

Cancer cell lines. Human pancreatic ductal epithelial cells (HPDE), pancreatic cancer cells BxPC-3, PANC-1, AsPC-1, HPAC, CFPAC-1, HS-766T, HPAF-II and SW1990 were obtained from Shanghai Institute for Life Science, Chinese Academy of Sciences. PANC-1 and HPAC were grown in $5 \% \mathrm{CO}_{2}$ saturated humidity, at $37^{\circ} \mathrm{C}$ and cultured in DMEM (Gibco, USA) supplemented with $2 \mathrm{mmol} / 1$ glutamine and $10 \%$ FBS (Gibco) and subcultured by harvesting with trypsin-EDTA. HPDE, BxPC-3, AsPC-1, CFPAC-1, HS-766T, HPAF-II and SW1990 cells were cultured in RPMI-1640 (Gibco) supplemented with 10\% FBS.

Knockdown and overexpression of the HDAC3 gene. Human HDAC3 cDNA (NM_003883) was amplified by RT-PCR with the RNA extracted from PANC-1 cells, and then cloned into pLV4 vector. HDAC3 were searched for suitable siRNA target sequences, three siRNA sequences were designed, synthesized, and confirmed by sequencing, respectively. Preliminary experiments picked out the most efficient siRNA sequences of knockdown from the above three candidate siRNAs. siRNA sequences: 5'-GCCUCAUCGCCUGGCAUUGdTdT-3' (sense), 5'-CAAUGCCAGGCGAUGAGGCdTdT-3' (antisense); with a negative control siRNA sequence: 5'-UUCUCCGAAC GUGUCACGUdTdT-3' (sense), 5'-ACGUGACACGUUCGGA GAAdTdT-3' (antisense). Lentivirus particles were produced by co-transfecting expression vector pLV4-HDAC3 cDNA, pLV4-HDAC3 shRNA or pLV4-vector with viral particle packaging helper vector into $293 \mathrm{~T}$ cells. Titer of viral particles was determined by limited serial dilution. PANC-1 cells or HPAC cells were infected with the lentivirus with the pLV4-HDAC3 cDNA, pLV4-HDAC3 shRNA or pLV4-vector. The efficiency of knockdown or overexpression of HDAC3 was determined by western blot analysis.

Cell proliferation assay and cytotoxicity studies. Cell proliferation was determined from three separate experiments using MTT (3-(4,5-dimethylthiazol-2-yl-2,5-diphenyltetrazolium bromide) assays (7). Briefly, the medium was removed and replaced by $200 \mu \mathrm{l}$ fresh medium with $500 \mu \mathrm{g} / \mathrm{ml}$ MTT per well. The cells were incubated for $4 \mathrm{~h}$. The medium was then removed and $200 \mu \mathrm{l}$ of DMSO was added to each well. The absorbance value of each well was determined spectrophotometrically at $570 \mathrm{~nm}$ on a Microplate ELISA Reader
(Bio-Tek Instruments). The cell growth inhibitory effect of 72-h gemcitabine exposure was studied as described previously (8). The $50 \%$ inhibitory concentration $\left(\mathrm{IC}_{50}\right)$ of cell growth for each cell line was determined by non-linear least squares curve fitting (GraphPad PRISM, Intuitive Software for Science). The experiment was performed in triplicate. Each experiment was repeated three times.

In vitro invasion assay and migration assay. MilliCell (12-mm diameter with $8-\mu \mathrm{m}$ pores) chambers (Millipore, Bedford, MA, USA) were pre-coated with Matrigel (BD, Bedford, MA, USA) on the upper side. A total of $1 \times 10^{5}$ pancreatic cancer cells were added to the upper compartment in medium supplemented with $0.1 \%$ serum, and the chambers were placed into 24 -well plates with medium containing $10 \%$ serum. After $24 \mathrm{~h}$ at $37^{\circ} \mathrm{C}$, cells on the upper side of the membrane were wiped off, invaded cells on the lower membrane surface were fixed and stained with DAPI (Sigma). Invasive activity was quantified by counting 10 high-power fields (HPFs, x200) per chamber. Mean values were obtained from at least three individual chambers for each experimental point per assay. The migration assay is the same with invasion assay excepting no Matrigel was used and the permeating time for cells was $16 \mathrm{~h}$.

Reverse transcription $q P C R$ analysis. Total RNA was extracted from the cells with RNAiso (Takara) according to the manufacturer's protocol. For mRNA detection, reverse transcription was performed according to the protocol of RevertAid $^{\mathrm{TM}}$ First Strand cDNA Synthesis kits (Fermentas); qPCR was performed with SYBR premix Ex Taq (Takara) on an Applied Biosystems 7500 Real-Time PCR system supplied with analytical software (Applied Biosystems, USA). GAPDH mRNA was used to normalize RNA inputs. Primers used were as follows: P27 [5'-GCAACCAATGGATCTCCT CCT-3' (sense) and 5'-GGGGAGAAAAACACCCCGAA-3' (antisense)], P53 [(5'-TGACACGCTTCCCTGGATTG-3' (sense) and 5'-TCCGGGGACAGCATCAAATC-3' (antisense)], Bax [(5'-GTGGTTGGGTGAGACTCCTC-3' (sense) and 5'-GCA GGGTAGATGAATCGGGG-3' (antisense)], Bim [(5'-TTGAT TCTTGCAGCCACCCT-3' (sense) and 5'-CGCAGGCTGC AATTGTCTAC-3' (antisense)], GAPDH [(5'-CTCTGCTCCT CCTGTTCGAC-3' (sense) and 5'-GCGCCCAATACGACCAA ATC-3' (antisense)]. Data were processed by using the $2^{-\Delta \Delta C t}$ method. The results are represented as the means \pm SE of three independent experiments.

Western blotting. Cells were lysed with lysis buffer (Beyotime) containing PMSF. Lysates was centrifuged at $12,000 \mathrm{~g}$ at $4^{\circ} \mathrm{C}$ for $10 \mathrm{~min}$, and the supernatant was collected and preserved at $-80^{\circ} \mathrm{C}$ for later use. Protein concentrations were determined using BCA Protein Assay kit (Beyotime). Proteins were subjected to one-dimensional SDS-PAGE and transferred to PVDF membrane (Millipore) by using a transfer apparatus according to the manufacturer's protocols (Bio-Rad, Richmond, CA, USA). Membranes were blocked with non-fat dry milk in TBST buffer $(10 \mathrm{mM}$ Tris- $\mathrm{HCl}, \mathrm{pH} 8.0$, and $150 \mathrm{mM} \mathrm{NaCl}$ ) containing $0.1 \%$ Tween-20, washed in the same buffer and probed with the following antibodies: anti-HDAC3 (Abcam), anti-histone H3 (Millipore Biotechnology), antiacetyl-histone H3 (Millipore Biotechnology) and anti- $\beta$-actin 
A
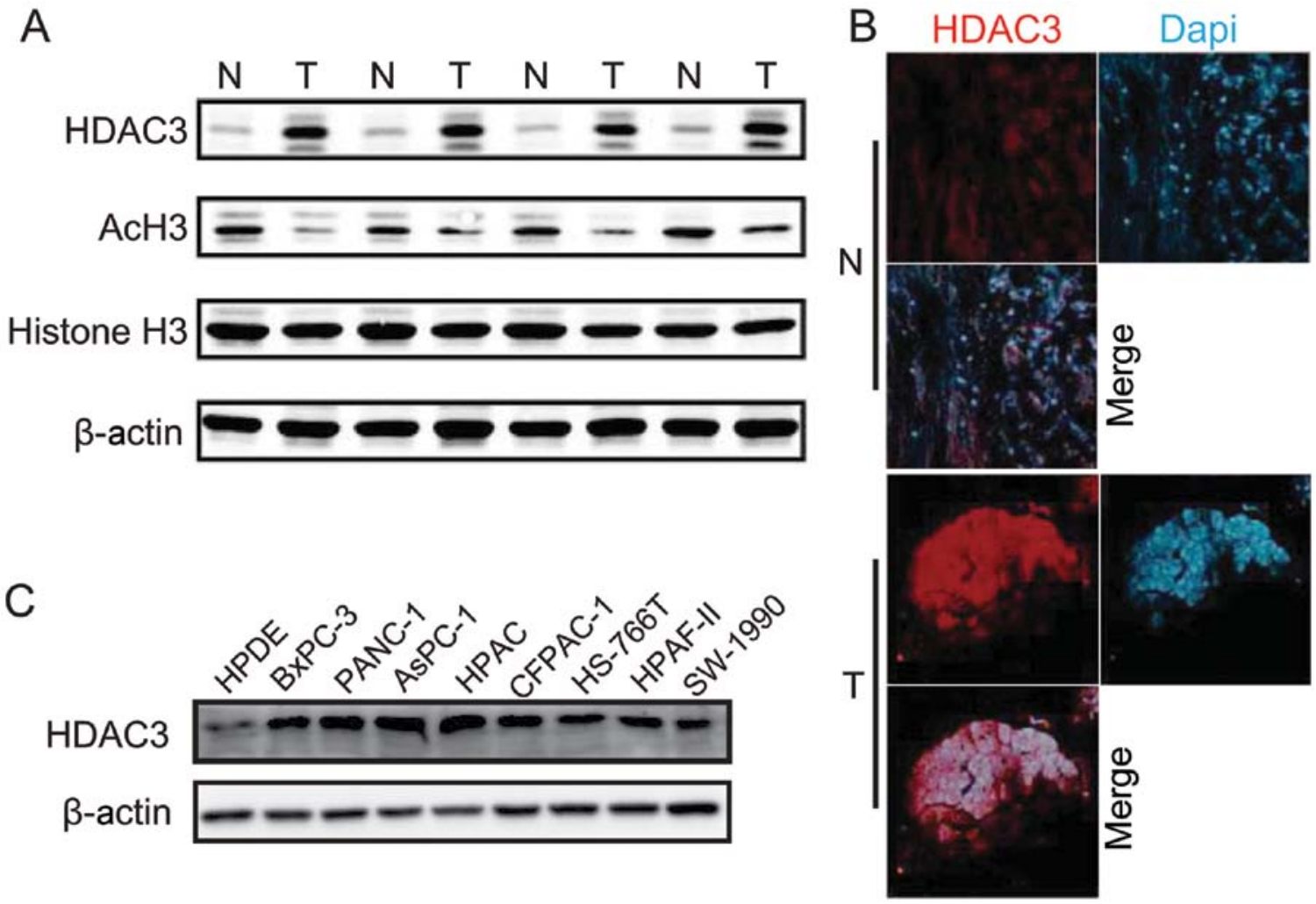

Figure 1. The levels of HDAC3 protein expression in pancreatic cancer. (A) HDAC3, histone $\mathrm{H} 3$ and acetylated histone $\mathrm{H} 3$ (AcH3) protein expression were detected by western blot analysis in tumor $(\mathrm{T})$ and paracancerous tissues (N) from pancreatic cancer patients. (B) HDAC3 protein expression was detected by immunofluorescence $(\mathrm{x} 40)$. (C) The protein levels of HDAC3 expression were compared between pancreatic cancer cell lines and human pancreatic ductal epithelial cells (HPDE).

antibodies (Cell Signaling Technology) at $4^{\circ} \mathrm{C}$ overnight. Then the membranes were washed in TBST buffer and incubated with respective secondary antibody. The infrared fluorescence image was obtained using Odyssey infrared imaging system (Li-Cor Bioscience). Data were obtained from at least three independent experiments.

Chromatin immunoprecipitation. Chromatin immunoprecipitation (ChIP) was performed using the Upstate Biotechnology ChIP kit and following a modified protocol from the manufacturer. Briefly, tissues was fixed in $1 \%$ formaldehyde and cell lysates were sheared by sonication in $1 \%$ SDS lysis buffer to generate chromatin fragments with an average length of 200-1,000 bp. The chromatin was then immunoprecipitated overnight at $4^{\circ} \mathrm{C}$ with antibodies specific to HDAC3 (Abcam), AcH3, AcH3K9 and AcH3K 14 (Millipore Biotechnology) or an equivalent amount of control IgG. Protein-DNA-antibody complexes were precipitated with protein A-agarose beads for $2 \mathrm{~h}$ at $4^{\circ} \mathrm{C}$. Input or DNA in the complex was subjected to quantitative PCR with specific primer for P27 promoter [(5'-GGCTCACAAGTTAGAGA CAA-3' (sense) and 5'-GCAGAAGGAATTAGCAAGTG-3' (antisense)],P53 promoter[(5'-CACAGGAACAGACGACAA-3' (sense) and 5'-TGGACACGGCTAAGTAGA-3' (antisense)], Bax promoter [(5'-TCTTACTATTGGTTGCTCTAGG-3' (sense) and 5'-AGGTCTCGGTTCTGTCTG-3' (antisense)], Bim promoter [(5'-CTTAGAAGAATGGTGGAGTTG-3' (sense) and 5'-CATAGACAAGTGTTCAGATGG-3' (antisense)].
Statistical analysis. All statistical analyses were performed using SPSS 10.0. Data are expressed as mean \pm SEM. The statistical correlation of data between groups was analyzed by one-way analysis of variance (ANOVA) and Student's t-test as appropriate. In vitro cell growth assay was tested using factorial design ANOVA. P-values of $<0.05$ were considered statistically significant.

\section{Results}

HDAC3 protein is overexpressed in human pancreatic cancer. Four samples of pancreatic cancer tissues and paired paracancerous tissues were collected. Protein levels of HDAC3 were detected by western blot analysis. In comparison with paired paracancerous tissues, HDAC 3 protein expression was significant higher in pancreatic cancer tissues. Next, we detected protein levels of histone $\mathrm{H} 3$ and acetylated histone $\mathrm{H} 3$ (AcH3). While total levels of histone $\mathrm{H} 3$ were similar, levels of $\mathrm{AcH} 3$ were significantly higher at paracarcinoma tissues as compared to tumor tissues (Fig. 1A). The results suggested that HDAC3 overexpression could lead to decreased level of AcH3 in pancreatic cancer tissues. In addition, immunofluorescence results confirmed that HDAC3 protein expression was higher in pancreatic cancer (Fig. 1B). Furthermore, we compared the expression of HDAC3 between human normal pancreatic ductal epithelial cell (HPDE) and pancreatic cancer cell lines. Interestingly, consistently higher expression level of HDAC3 was found in all the eight pancreatic cancer cell lines relative to the HPDE (Fig. 1C). Taken together, it is suggested that 
A

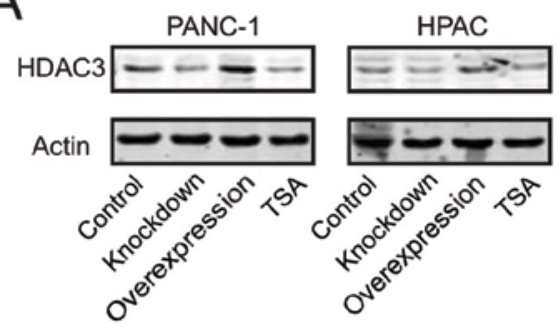

C
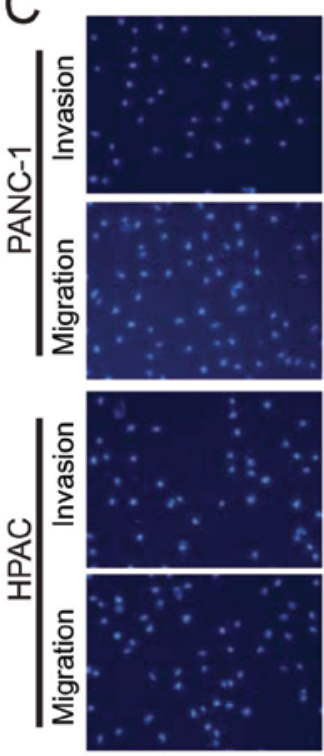

Control
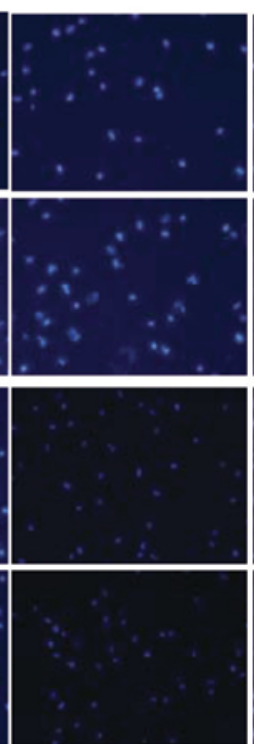

B
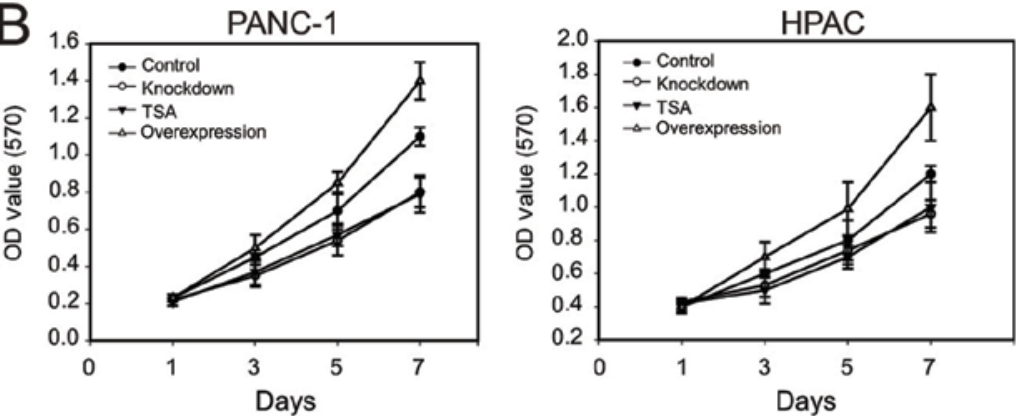

口Control घKnockdown $\square$ Overexpression QTSA
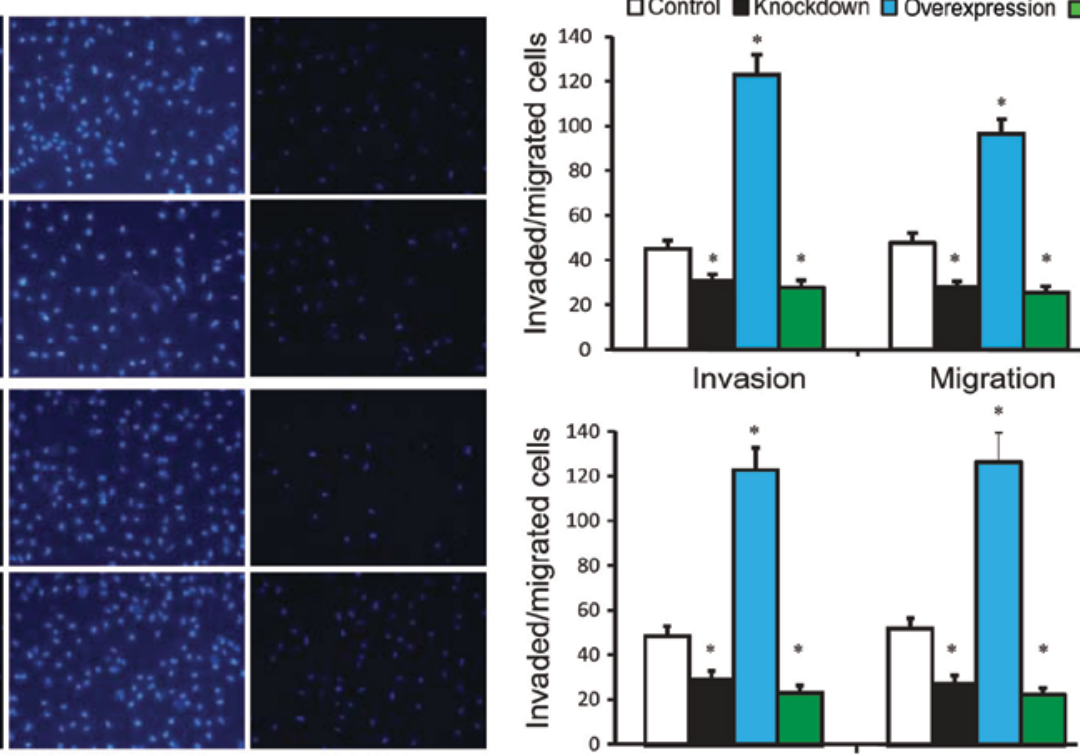

TSA

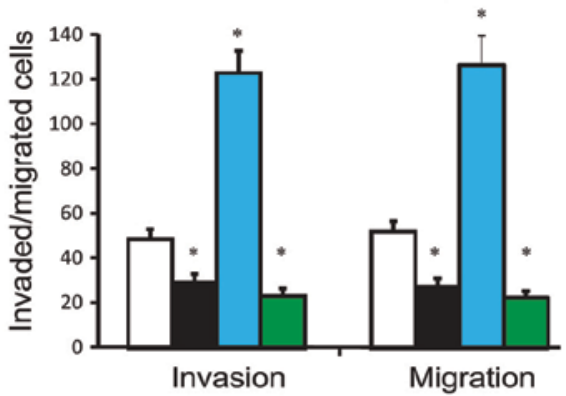

D
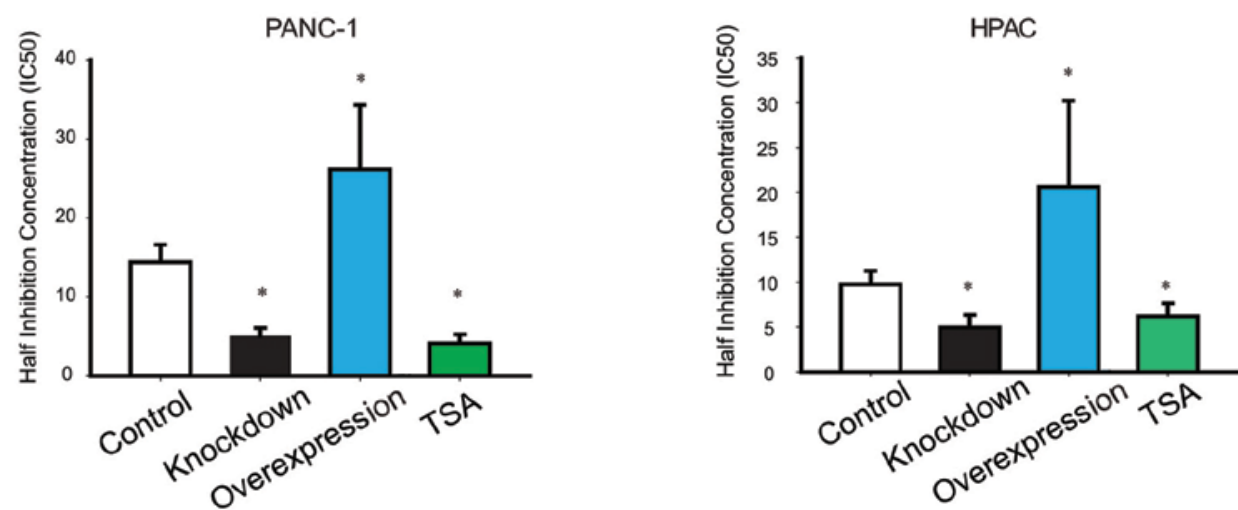

Figure 2. Effects of HDAC3 expression on pancreatic cancer cell proliferation, migration and invasion, and drug resistance in vitro. (A) Protein expression of HDAC3 was detected in HDAC3-knockdown and HDAC3-overpression stable transfected cells, TSA treatment and negative control. (B) In vitro cell proliferation was analyzed by MTT assay $(\mathrm{P}<0.05)$. (C) Capabilities of migration and invasion were analyzed by Transwell assay (migration, without Matrigel; invasion, coated by Matrigel). Values represent the number of migratory/invasive cells per 5 high power fields (x200). (D) The $50 \%$ inhibitory concentration $\left(\mathrm{IC}_{50}\right)$ of gemcitabine was compared. Data are representative or expressed as the mean \pm SEM of three independent experiments. ${ }^{*} \mathrm{P}<0.05$ or ${ }^{* *} \mathrm{P}<0.01$, compared with control group.

upregulation of HDAC3 is a frequent event in human pancreatic cancer.

HDAC3 promotes pancreatic cancer cell proliferation, migration and invasion, and increases drug resistance in vitro. In order to investigate the biological function involved in pancreatic cancer, we knocked down or overexpressed
HDAC3 gene expression in PANC-1 and HPAC cells through lentivirus-mediated constructs. In addition, TSA, an inhibitor of HDACs, was used to inhibit HDAC3 activity. As shown in Fig. 2A, comparing to the control, cells transfected with shRNA-HDAC3 or treatment with TSA had decreased levels of HDAC3 protein, and HDAC3 overexpression was able to upregulate HDAC3 protein level significantly. 
A

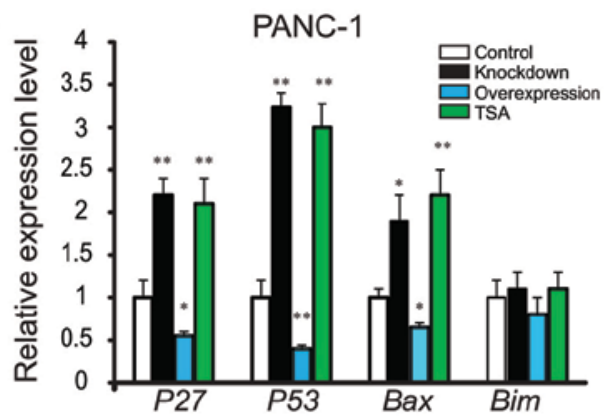

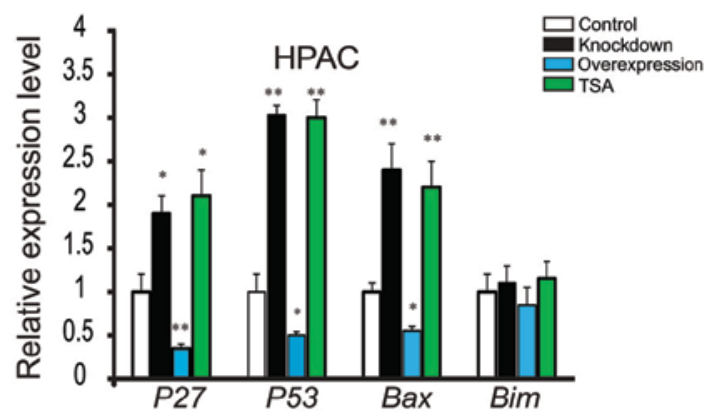

B
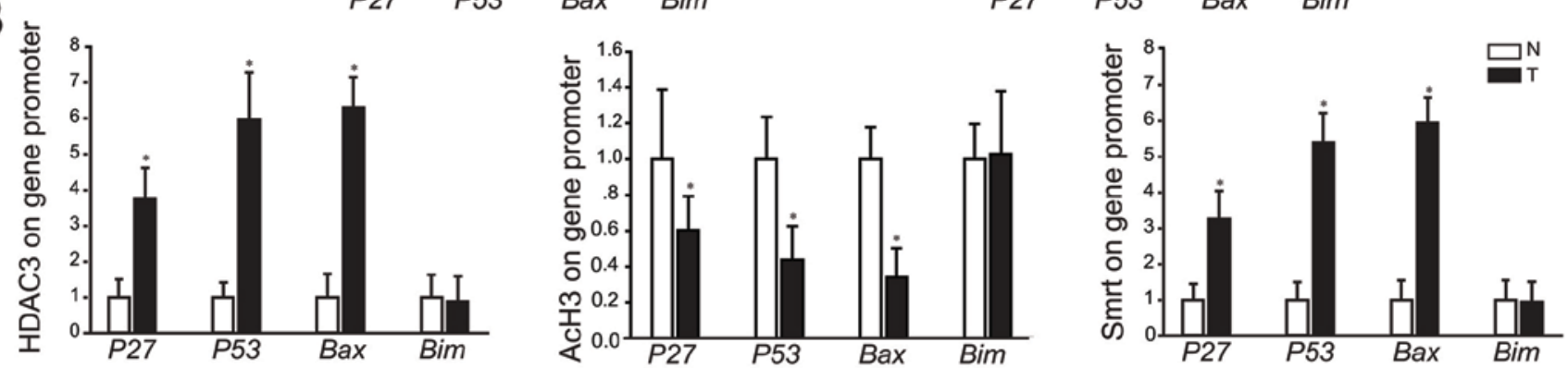

C
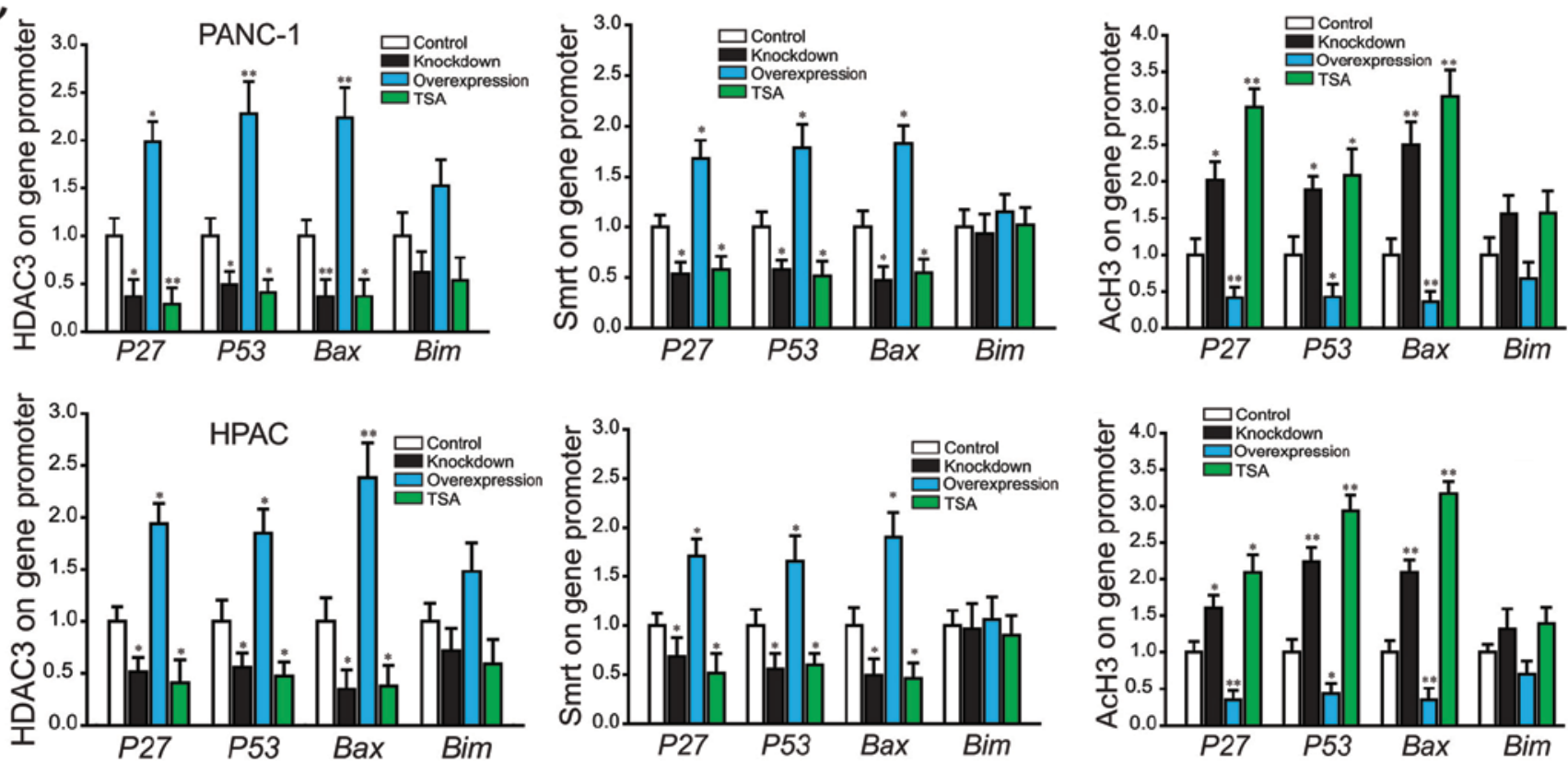

Figure 3. Effects of HDAC3 expression on gene transcriptional regulation. (A) Reverse transcription qPCR analysis of P53, P27, Bax and Bim mRNA expression. (B) The recruitment levels of HDAC3, AcH3 and SMRT to P27, P53, Bax and Bim gene promoter were determined by ChIP assay in pancreatic cancer (T) and paracancerous tissues (N). (C) The levels of HDAC3, AcH3 and SMRT recruited to P27, P53, Bax and Bim gene promoter were affected by HDAC 3 expression in pancreatic cancer cell lines PANC-1 and HPAC. Data are expressed as the mean \pm SEM of three independent experiments. ${ }^{*}<0.05$ or ${ }^{* *} \mathrm{P}<0.01$, compared with control group.

To detect the effect of HDAC3 expression on pancreatic cell proliferation, we investigated the proliferative activities by MTT assays. As a result, it was found that HDAC3 downregulation significantly reduced the proliferative activities of pancreatic cancer cell lines, while HDAC3 upregulation increased the cell proliferation (Fig. 2B).

Cell migration and invasion are important processes of tumor development and metastasis. Therefore, we evaluated the effects of HDAC3 on the migration and invasion of PANC-1 and HPAC by the Transwell assay. The results revealed that migration/invasion capability of PANC-1 and
HPAC cells were significantly decreased by HDAC3 knockdown or treatment with TSA, and increased by HDAC3 upregulation (Fig. 2C).

To screen the effect of HDAC3 on drug resistance, we investigated the impact gemcitabine on the proliferation of PANC-1 and HPAC cells, and calculated the half inhibition concentration $\left(\mathrm{IC}_{50}\right)$. The results are shown in Fig. 2D, compared to control, HDAC3 knockdown or TSA treatment decreased $\mathrm{IC}_{50}$ of gemcitabine (PANC-1, 5.04 $\pm 1.04 / 4.11 \pm 1.14$ vs. $14.40 \pm 2.21 \mathrm{nmol} / 1$; HPAC, $4.93 \pm 1.41 / 6.17 \pm 1.48$ vs. $9.74 \pm 1.52 \mathrm{nmol} / \mathrm{l})$, and HDAC3 overexpression increased 

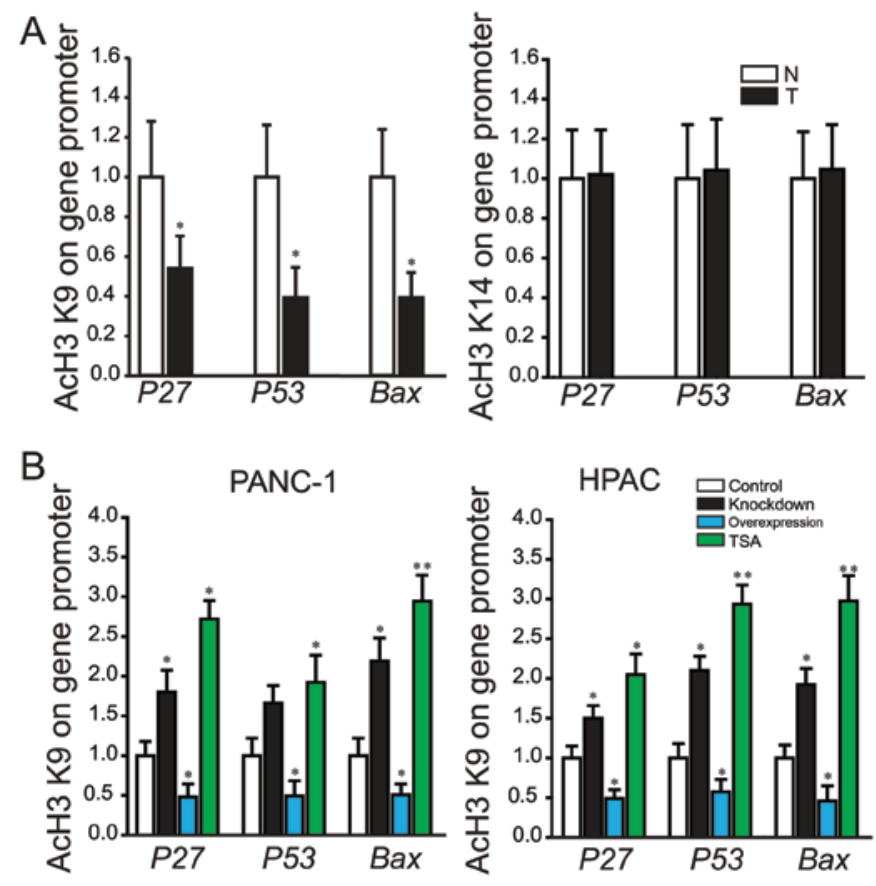

Figure 4. The recruitment of HDAC3 to gene promoter mediates transcriptional repression via $\mathrm{H} 3 \mathrm{~K} 9$ deacetylation. (A) The modulation of histone $\mathrm{H} 3 \mathrm{~K} 9$ and $\mathrm{H} 3 \mathrm{~K} 14$ acetylation at the P27, P53, Bax and Bim promoters were examined by ChIP assay in pancreatic cancer $(\mathrm{T})$ and paracancerous tissues (N). (B) The recruitment levels of histone H3K9 acetylation to P27, P53 and Bax gene promoter were affected by HDAC3 expression in pancreatic cancer cell lines PANC-1 and HPAC. Data are expressed as the mean \pm SEM of three independent experiments. ${ }^{*} \mathrm{P}<0.05$ or ${ }^{* *} \mathrm{P}<0.01$, compared with control group.

$\mathrm{IC}_{50}$ significantly (PANC-1, 26.13 \pm 8.16 vs. $14.40 \pm 2.21 \mathrm{nmol} / \mathrm{l}$; HPAC, $20.62 \pm 9.61$ vs. $9.74 \pm 1.52 \mathrm{nmol} / \mathrm{l})$.

The recruitment of high level of HDAC3 to P27, P53 and Bax gene promoter mediate transcription suppression. Elevated levels of HDACs expression among malignancies caused transcriptional repression of a diverse set of genes, involved in tumor progression (5). Previous reports suggested that P27, P53, Bax and Bim played important roles in pancreatic cancer progression. The P53 tumor suppressor gene encodes a nuclear protein that plays a crucial role in cell cycle regulation and major early events in pancreatic cancer (9). P27 (Kip1/CDKN1B) is a member of the Cip/Kip family of cyclin-dependent kinase inhibitors, which can induce cell cycle arrest and serve as tumor suppressors. Moreover, P27 and P53 provide independent prognostic or predictive information in pancreatic cancer (10). Bax and Bim belong to the BCL-2 family forming hetero- or homodimers and acting as anti- or pro-apoptotic regulators that are involved in a wide variety of cellular activities. Bax (11) and Bim (12) function as an apoptotic activator regulated apoptosis signaling in pancreatic cancer. To investigate the possible mechanisms of HDAC3-mediated biological function in pancreatic cancer, we analysed the effects of HDAC3 expression on P27, P53, Bax and Bim mRNA. The results revealed that expression of P27, P53 and Bax mRNA was upregulated by HDAC3 knockdown, and significantly downregulated by HDAC3 overexpression. However, no changes of the expression level of Bim gene mRNA was observed (Fig. 3A). Therefore, we hypothesized that recruitment of high level of HDAC 3 to the above gene promoters in pancreatic cancer led to transcription suppression. The ChIP result revealed that, compared to paired paracancerous tissues, higher level of HDAC3 was recruited to $\mathrm{P} 27, \mathrm{P} 53$ and Bax promoter in pancreatic cancer. In addition, there was no statistically significant difference for Bim gene. Accordingly, the recruitment of $\mathrm{AcH} 3$ was significantly diminished because of the higher level of HDAC3 (Fig. 3B).

Specifically, HDAC3 negatively regulates the transcription of genes by directly interacting with co-repressors recruited to target gene promoters by various transcription factors (13). The silencing mediator of retinoid and thyroid receptor (SMRT) is a nuclear receptor corepressor that binds and enhances the HDAC activity of HDAC3 (14). ChIP experiments with SMRT antibodies indicated increased recruitment in the P27, P53 and Bax promoter region in pancreatic cancer (Fig. 3B), thus confirming a functional involvement of HDAC3 in the postinduction repression of gene transcription.

Furthermore, the recruitments of HDAC3 and SMRT to the promoters of P27, P53 and Bax gene were significantly diminished by knockdown of HDAC 3 or TSA administration, and increased by HDAC3 overexpression. Inversely, HDAC3 knockdown or treatment with TSA could increase the recruitment of $\mathrm{AcH} 3$, and $\mathrm{HDAC} 3$ overexpression resulted in decreased level of $\mathrm{AcH} 3$ recruitment (Fig. 3C).

\section{H3K9 deacetylation is directly correlated with the recruitment} of HDAC3 to P27, P53 and Bax gene promoters. Genomewide analyses of histone acetylation have demonstrated that the acetylation of individual lysines in histone $\mathrm{H} 3$ and $\mathrm{H} 4$ tails and more specifically histone $\mathrm{H} 3$ acetylation at lysines $\mathrm{K} 9$

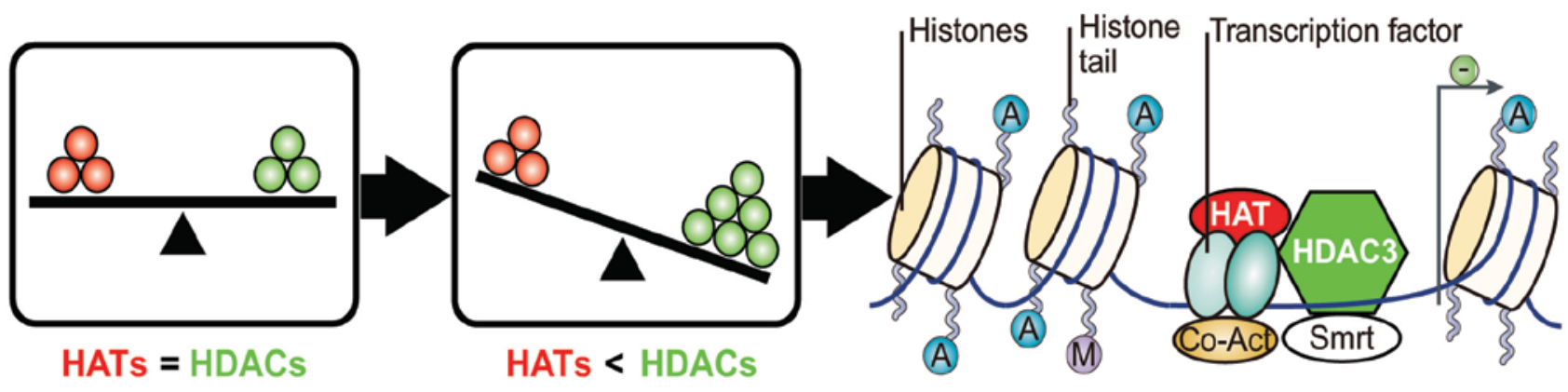

Figure 5. Proposed model of HDAC3 in regulation of the repression of gene transcription. HATs, histone acetylases; HDACs, histone deacetylases. 
(H3K9) and K14 (H3K14) correlates with the active state of gene transcription $(15,16)$. To further understand the involvement of histone acetylation in regulating gene expression, the modulation of histone $\mathrm{H} 3 \mathrm{~K} 9$ and $\mathrm{H} 3 \mathrm{~K} 14$ acetylation at the P27, P53 and Bax promoters were examined. Compared to the constitutive levels detected in paired paracancerous tissues, decreased $\mathrm{H} 3 \mathrm{~K} 9$ acetylation by 2-3-fold was detected in pancreatic cancer tissues (Fig. 4A). However, for H3K14 acetylation, no significant difference was detected (Fig. 4A). These results indicated $\mathrm{H} 3 \mathrm{~K} 9$ deacetylation correlates directly with the recruitment of HDAC3 to the above gene promoters, thus providing a mechanism to maintain controlled expression of genes.

Furthermore, ChIP assay was carried out to determine the levels of $\mathrm{H} 3 \mathrm{~K} 9$ acetylation at the gene promoter in PANC-1 and HPAC with stable transfection of HDAC3 or siRNA-HDAC3. The recruitment levels of $\mathrm{H} 3 \mathrm{~K} 9$ acetylation were significantly diminished by HDAC3 overexpression, and increased by knockdown of HDAC3 or treatment with TSA (Fig. 4B).

Taken together, these results indicated that recruitment of HDAC3 to P27, P53 and Bax gene promoters led to histone $\mathrm{H} 3 \mathrm{~K} 9$ deacetylation and post-inductional inhibition of gene transcription in pancreatic cancer.

\section{Discussion}

Within tumor cells, aberrant deacetylation of histones due to enhanced HDACs activity results in conformational changes within the nucleosome that lead to transcriptional repression of genes involved in differentiation and negative regulation of cell proliferation, invasion and metastasis (Fig. 5). Increased expressions of HDAC3 have been found closely associated with many malignancies (5). In several studies analyzing patient cancer samples, overexpression of HDAC3 was found in hepatocellular (17), lung (18) and prostate carcinomas (19) and in most of the cases HDAC 3 upregulation associates with poor prognosis. Also it was proposed to serve as a candidate biomarker (20). However, the contributions of HDAC3 to pancreatic cancer remain incompletely understood.

In the present study, we firstly analyzed HDAC 3 expression in pancreatic cancer and paired paracancerous tissues as a control. The result showed higher HDAC 3 expression in pancreatic cancer tissues. Consistently, all the eight pancreatic cancer cell lines had high level of HDAC3. In addition, our data suggested that elevated expression of HDAC3 in pancreatic cancer led to reduced level of histone $\mathrm{H} 3$ acetylation. To the best of our knowledge, this is the first report that demonstrates higher level of HDAC3 expression in pancreatic cancer.

Although we demonstrated that HDAC3 functioned as a stimulus in pancreatic cancer, the function of HDAC3 in tumor is still very controversial. Various researchers have viewed HDAC3 as an oncogenic protein. SiRNA-mediated knock-down of HDAC3 in HeLa cells resulted in inhibition of cell proliferation (21). Inhibition of HDAC3 in glioma cell lines could suppress proliferation and tumor sphere formation, induce G0/G1 arrest and apoptosis, and suppress the migration of glioma cells in comparison with controls (22). However, it has also been reported as a tumor suppressor protein (23). To further understand the biologic function of HDAC3, pancreatic cancer cell lines PANC-1 and HPAC with stable overexpression and knockdown HDAC3 gene were constructed by lentivirus-mediated methods. Our data showed that knock-down of HDAC3 expression could suppress pancreatic cancer cell proliferation, migration and invasion, and reduce drug-resistance of gemcitabine, consistent with TSA administration, an inhibitor of HDAC(s). Accordingly, HDAC3 overexpression promoted cell proliferation, migration and invasion, and increased drug-resistance. Our study revealed that HDAC3 may function as an oncogenic protein in pancreatic cancer cells, which was in agreement with previous findings in other cell types $(22,24)$. Together, these results suggested that the role of HDAC3 in pancreatic cancer may mainly lie in tumor growth, invasion and metastasis.

Previous studies suggested that P27, P53, Bax and Bim played major roles in pancreatic cancer progression, which could be regulated by HDAC and/or histone deacetylase inhibitors (HDACi). HDACi activated P53, and entinostatinduced cytotoxic effects partially depended on P53 in colon cancer cell lines (25). Valproic acid and TSA affect acetylation status of p53 and induce apoptosis in ERG-positive prostate cancer cells (26). In human lung cancer cell lines, HDAC inactivation results in the induction of apoptosis via p53 and Bax activation (27). P27, which inhibits CDK4- and CDK2-containing complexes, was induced by vorinostat and/or TSA, in leukemia cells (28) and breast cancer cells (29). In addition, HDACi could upregulate pro-apoptotic proteins of Bcl-2 family, such as Bax, Bim, Bmf, Bak and Bik $(30,31)$, which mediated the intrinsic apoptosis pathway. Moreover, HDAC3 played a role in cell cycle processes and DNA damage response (32). Therefore, we hypothesized the functional involvement of HDAC3 in pancreatic cancer were possibly correlated with the post-induction repression of gene transcription, including P27, P53, Bax and Bim. Firstly, our results showed that P27, P53 and Bax mRNA expressions were upregulated by HDAC3 knockdown, and downregulated significantly by HDAC3 overexpression. Further ChIP analysis revealed that, compared to paracancerous tissues, higher level of HDAC3 and HDAC3 corepressors SMRT were recruited to P27, P53 and Bax promoter in pancreatic cancer, leading to the decreased $\mathrm{AcH} 3$ at gene promoter. In regulation of Bim gene expression, other mechanisms may also exist. Together, the above data suggest that the functional involvements of HDAC3 in pancreatic cancer were possible partially associated with the transcription repression of P27, P53 and Bax genes. However, the detail mechanisms of HDAC3 regulating histone modifications and key factors in pancreatic cancer still need further exploration.

Furthermore, histone $\mathrm{H} 3$ acetylation at lysines K9 (H3K9) and K14 (H3K14) were directly correlated with the active state of gene transcription (16). In order to identify which lysines acetylation/deacetylation of histone H3 was responsible for regulating genes expression, ChIP assays with $\mathrm{H} 3 \mathrm{~K} 9$ and H3K14 antibodies were performed. The results indicated that inhibition of P27, P53 and Bax gene transcription in pancreatic cancer were related to inhibition of histone $\mathrm{H} 3 \mathrm{~K} 9$ acetylation in the gene promoters. Knockdown of HDAC3 expression or treatment with TSA resulted in increase of H3K9 acetylation, whereas the levels of H3K14 acetylation were unaffected. Accordingly, HDAC3 overexpression led to decrease of $\mathrm{H} 3 \mathrm{~K} 9$ acetylation. Taken together, the functional 
involvement of HDAC3 in the post-induction repression of regulated gene transcription, were directly correlated to histone $\mathrm{H} 3 \mathrm{~K} 9$ deacetylation.

In conclusion, our findings reveal strong expression of HDAC 3 in patients with pancreatic cancer, and demonstrate that the functional involvement of HDAC3 is partially related to post-induction repression of P53, P27 and Bax gene transcription, acting via $\mathrm{H} 3 \mathrm{~K} 9$ deacetylation. HDAC3 participates in the pathogenesis and progression of pancreatic cancer, which might be a pivotal epigenetic target against this devastating disease.

\section{Acknowledgements}

This study was supported by the Natural Science Foundation of China (grant nos. 81101846, 81171887, 91229117 and 31101016), Program of Shanghai Subject Chief Scientist (grant no. 12XD1404200), Shanghai International Science and Technology Cooperation Project (grant no. 12410709000) and Shanghai Science and Technology Committee (grant no. 11DZ1922002).

\section{References}

1. Ducreux M, Boige V, Goere D, Deutsch E, Ezra P, Elias D and Malka D: The multidisciplinary management of gastrointestinal cancer. Pancreatic cancer: from pathogenesis to cure. Best Pract Res Clin Gastroenterol 21: 997-1014, 2007.

2. Ghaneh P, Costello E and Neoptolemos JP: Biology and management of pancreatic cancer. Postgrad Med J 84: 478-497, 2008.

3. Chueh A C, Togel L, Mariadason J and Tse JW: Mechanisms of HDAC inhibitor-regulated gene expression in cancer cells Antioxid Redox Signal March 27, 2014 [Epub ahead of print].

4. West AC and Johnstone RW: New and emerging HDAC inhibitors for cancer treatment. J Clin Invest 124: 30-39, 2014.

5. Barneda-Zahonero B and Parra M: Histone deacetylases and cancer. Mol Oncol 6: 579-589, 2012.

6. Huang X, Ji G, Wu Y, Wan B and Yu L: LAMA4, highly expressed in human hepatocellular carcinoma from Chinese patients, is a novel marker of tumor invasion and metastasis. J Cancer Res Clin Oncol 134: 705-714, 2008.

7. Xue Y, Ren H, Xiao W, Chu Z, Lee JJ and Mao L: Antitumor activity of AZ64 via G2/M arrest in non-small cell lung cancer. Int J Oncol 41: 1798-1808, 2012.

8. Giovannetti E, Del TM, Mey V, Funel N, Nannizzi S, Ricci S, Orlandini C, Boggi U, Campani D, Del CM, Iannopollo M, Bevilacqua G, Mosca F and Danesi R: Transcription analysis of human equilibrative nucleoside transporter-1 predicts survival in pancreas cancer patients treated with gemcitabine. Cancer Res 66: 3928-3935, 2006.

9. Bardeesy N, Aguirre AJ, Chu GC, Cheng KH, Lopez LV, Hezel AF, Feng B, Brennan C, Weissleder R, Mahmood U, Hanahan D, Redston MS, Chin L and Depinho RA: Both p16(Ink4a) and the p19(Arf)-p53 pathway constrain progression of pancreatic adenocarcinoma in the mouse. Proc Natl Acad Sci USA 103: 5947-5952, 2006.

10. Ansari D, Rosendahl A, Elebro J and Andersson R: Systematic review of immunohistochemical biomarkers to identify prognostic subgroups of patients with pancreatic cancer. Br J Surg 98: $1041-1055,2011$

11. Hamada S, Masamune A, Miura S, Satoh K and Shimosegawa T: MiR-365 induces gemcitabine resistance in pancreatic cancer cells by targeting the adaptor protein $\mathrm{SHC1}$ and pro-apoptotic regulator BAX. Cell Signal 26: 179-185, 2014.

12. Chen Z, Chen L Y, Dai H Y, Wang P, Gao S and Wang K: miR-301a promotes pancreatic cancer cell proliferation by directly inhibiting Bim expression. J Cell Biochem 113: 3229-3235, 2012.

13. Genin P, Lin R, Hiscott J and Civas A: Recruitment of histone deacetylase 3 to the interferon-A gene promoters attenuates interferon expression. PLoS One 7: e38336, 2012.

14. Karagianni P and Wong J: HDAC3: taking the SMRT-N-CoRrect road to repression. Oncogene 26: 5439-5449, 2007.
15. Pokholok DK, Harbison CT, Levine S, Cole M, Hannett NM, Lee TI, Bell GW, Walker K, Rolfe PA, Herbolsheimer E, Zeitlinger J, Lewitter F, Gifford DK and Young RA: Genomewide map of nucleosome acetylation and methylation in yeast. Cell 122: 517-527, 2005

16. Guenther M G, Levine SS, Boyer LA, Jaenisch R and Young RA: A chromatin landmark and transcription initiation at most promoters in human cells. Cell 130: 77-88, 2007.

17. Liu C, Liu L, Shan J, Shen J, Xu Y, Zhang Q, Yang Z, Wu L, Xia F, Bie P, Cui Y, Zhang X, Bian X and Qian C: Histone deacetylase 3 participates in self-renewal of liver cancer stem cells through histone modification. Cancer Lett 339: 60-69, 2013.

18. Minamiya Y, Ono T, Saito H, Takahashi N, Ito M, Motoyama S and Ogawa J: Strong expression of HDAC3 correlates with a poor prognosis in patients with adenocarcinoma of the lung. Tumour Biol 31: 533-539, 2010.

19. Weichert W, Roske A, Gekeler V, Beckers T, Stephan C, Jung K, Fritzsche F R, Niesporek S, Denkert C, Dietel M and Kristiansen G: Histone deacetylases 1,2 and 3 are highly expressed in prostate cancer and HDAC2 expression is associated with shorter PSA relapse time after radical prostatectomy. Br J Cancer 98: 604-610, 2008.

20. Wu LM, Yang Z, Zhou L, Zhang F, Xie H Y, Feng XW, Wu J and Zheng SS: Identification of histone deacetylase 3 as a biomarker for tumor recurrence following liver transplantation in HBV-associated hepatocellular carcinoma. PLoS One 5: e14460, 2010.

21. Glaser KB, Li J, Staver MJ, Wei RQ, Albert DH and Davidsen SK: Role of class I and class II histone deacetylases in carcinoma cells using siRNA. Biochem Biophys Res Commun 310: 529-536, 2003.

22. Zhu J, Wan H, Xue C, Jiang T, Qian C and Zhang Y: Histone deacetylase 3 implicated in the pathogenesis of children glioma by promoting glioma cell proliferation and migration. Brain Res 1520: 15-22, 2013.

23. Campos B, Bermejo J L, Han L, Felsberg J, Ahmadi R, Grabe N, Reifenberger G, Unterberg A and Herold-Mende C: Expression of nuclear receptor corepressors and class I histone deacetylases in astrocytic gliomas. Cancer Sci 102: 387-392, 2011.

24. Spurling CC, Godman CA, Noonan EJ, Rasmussen TP, Rosenberg DW and Giardina C: HDAC3 overexpression and colon cancer cell proliferation and differentiation. Mol Carcinog 47: 137-147, 2008.

25. Sonnemann J, Marx C, Becker S, Wittig S, Palani CD, Kramer $\mathrm{OH}$ and Beck J F: p53-dependent and p53-independent anticancer effects of different histone deacetylase inhibitors. Br J Cancer 110: 656-667, 2014.

26. Fortson WS, Kayarthodi S, Fujimura Y, Xu H, Matthews R, Grizzle WE, Rao VN, Bhat GK and Reddy ES: Histone deacetylase inhibitors, valproic acid and trichostatin-A induce apoptosis and affect acetylation status of p53 in ERG-positive prostate cancer cells. Int J Oncol 39: 111-119, 2011.

27. Jung KH, Noh JH, Kim JK, Eun JW, Bae HJ, Xie HJ, Chang YG, Kim MG, Park H, Lee JY and Nam SW: HDAC2 overexpression confers oncogenic potential to human lung cancer cells by deregulating expression of apoptosis and cell cycle proteins. J Cell Biochem 113: 2167-2177, 2012.

28. Nimmanapalli R, Fuino L, Stobaugh C, Richon V and Bhalla K: Cotreatment with the histone deacetylase inhibitor suberoylanilide hydroxamic acid (SAHA) enhances imatinib-induced apoptosis of Bcr-Abl-positive human acute leukemia cells. Blood 101: 3236-3239, 2003.

29. Huang L and Pardee AB: Suberoylanilide hydroxamic acid as a potential therapeutic agent for human breast cancer treatment. Mol Med 6: 849-866, 2000.

30. Zhao Y, Tan J, Zhuang L, Jiang X, Liu ET and Yu Q: Inhibitors of histone deacetylases target the Rb-E2F1 pathway for apoptosis induction through activation of proapoptotic protein Bim. Proc Natl Acad Sci USA 102: 16090-16095, 2005.

31. Xu W, Ngo L, Perez G, Dokmanovic M and Marks PA: Intrinsic apoptotic and thioredoxin pathways in human prostate cancer cell response to histone deacetylase inhibitor. Proc Natl Acad Sci USA 103: 15540-15545, 2006.

32. Reichert N, Choukrallah MA and Matthias P: Multiple roles of class I HDACs in proliferation, differentiation, and development. Cell Mol Life Sci 69: 2173-2187, 2012. 